\title{
Systematic study of isoscaling behavior in projectile fragmentation by the statistical abrasion-ablation model
}

\author{
D. Q. Fang $\$$, Y. G. Ma, C. Zhong, C. W. Ma, X. Z. Cai, J. G. \\ Chen, W. Guo, Q. M. Su, W. D. Tian, K. Wang, T. Z. Yan, W. \\ Q. Shen \\ Shanghai Institute of Applied Physics, Chinese Academy of Sciences, Shanghai \\ 201800, People's Republic of China
}

\begin{abstract}
The isospin effect and isoscaling behavior in projectile fragmentation have been systematically investigated by a modified statistical abrasion-ablation (SAA) model. The normalized peak differences and reduced isoscaling parameters are found to decrease with $\left(Z_{\text {proj }}-Z\right) / Z_{\text {proj }}$ or the excitation energy per nucleon and have no significant dependence on the size of reaction systems. Assuming a Fermi-gas behavior, the excitation energy dependence of the symmetry energy coefficients are tentatively extracted from $\alpha$ and $\beta$ which looks consistent with the experimental data. It is pointed out that the reduced isoscaling parameters can be used as an observable to study excitation extent of system and asymmetric nuclear equation of state in heavy ion collisions.

PACS numbers: 25.70.Mn, 24.10.Pa
\end{abstract}

\section{Introduction}

The process of projectile fragmentation has been studied extensively for investigation of reaction mechanisms in heavy ion collisions at intermediate and high energies [1, 2, 3, 4, 5, 6, 7]. It is also one of the most important methods to produce exotic nuclei. Recent advances in experiments using radioactive ion beams with large neutron or proton excess have lead to the discovery of halo structure [8, 9]. Since then interest in the study of very neutron-rich and proton-rich nuclei has grown due to their anomalous structures. In addition, the studies of isospin physics have become a very popular subject. The isospin effects of various physical phenomena, such as multifragmentation, flow, pre-equilibrium nucleon emission, etc., have been extensively reported [10, 11, 12, 13, 14, 15, 16, 17]. The studies have shown that isospin effect exists in nuclear reactions induced by exotic nuclei but it may disappear under certain conditions. Our previous calculations by using the modified statistical abrasion-ablation (SAA) model have demonstrated that the fragment isotopic distribution shifts toward

$\ddagger$ Corresponding author. Email address: dqfang@sinap.ac.cn 
the neutron-rich side for neutron-rich projectile, but the shift decreases with the increase of the parameter $\left(Z_{\text {proj }}-\mathrm{Z}\right) / Z_{\text {proj }}$ or the violence of nuclear reaction. This isospin effect of fragmentation reaction on the fragment isotopic distribution will disappear when $\left(\mathrm{Z}_{\text {proj }^{-Z}} \mathrm{Z}\right) / \mathrm{Z}_{\text {proj }}$ becomes larger than 0.5 [18, 19].

Recently, study of the nuclear symmetry energy has become a very important topic in nuclear physics. It is well known that the nuclear symmetry energy is very significant for investigation of the nuclear equation of state and a variety of astrophysical phenomena. The isoscaling approach for light fragment composition produced in the multifragmentation of very hot source has become an important method in heavy ion collisions since it can isolate the nuclear symmetry energy in the fragment yields [20, 21, 22, 23]. The scaling law relates ratios of isotope yields measured in two different nuclear reactions, 1 and $2, R_{21}(N, Z)=Y_{2}(N, Z) / Y_{1}(N, Z)$. In multifragmentation events, such ratios are shown to obey an exponential dependence on the neutron number $N$ or proton number $Z$ of the isotopes or isotones characterized by three parameters $\alpha$, $\beta$ and $\mathrm{C}[20]$ :

$$
R_{21}(N, Z)=\frac{Y_{2}(N, Z)}{Y_{1}(N, Z)}=C \exp (\alpha N+\beta Z),
$$

here $C$ is an overall normalization constant. In the grand-canonical limit, $\alpha$ and $\beta$ will have the form,

$$
\alpha=\frac{4 C_{\mathrm{Sym}}}{T}\left[\left(\frac{Z_{1}}{A_{1}}\right)^{2}-\left(\frac{Z_{2}}{A_{2}}\right)^{2}\right] \equiv \frac{4 C_{\mathrm{Sym}}}{T} \Delta\left[\left(\frac{Z}{A}\right)^{2}\right]
$$

and

$$
\beta=\frac{4 C_{\mathrm{Sym}}}{T}\left[\left(\frac{N_{1}}{A_{1}}\right)^{2}-\left(\frac{N_{2}}{A_{2}}\right)^{2}\right] \equiv \frac{4 C_{\mathrm{Sym}}}{T} \Delta\left[\left(\frac{N}{A}\right)^{2}\right],
$$

where $C_{\text {Sym }}$ is symmetry energy coefficient $(\mathrm{MeV}),\left(\frac{Z_{\mathrm{i}}}{A_{\mathrm{i}}}\right)^{2}$ or $\left(\frac{N_{\mathrm{i}}}{A_{\mathrm{i}}}\right)^{2}(\mathrm{i}=1,2)$ means the square of charge or neutron number over mass number for system 1 and 2. $T$ is the temperature of the system in $\mathrm{MeV}$. This behavior is attributed to the difference of isospin asymmetry between two reaction systems in similar nuclear temperature. Since the symmetry energy determines nuclear structure of neutron-rich or neutron-deficient rare isotopes, studies on the isoscaling behavior can be used to probe the isospin dependent nuclear equation of state [20, 21, 22, 23, 24, 25, 26, 27, 28, 29].

So far, the isoscaling behavior has been studied experimentally and theoretically for different reaction mechanisms. However, most studies focus on the isoscaling behaviors for light particles with $Z=2-8$. A few studies on the heavy projectile-like residues in deep elastic collisions and fission fragments have been reported [29, 30, 31, 32, 33, 34].

In this paper, we will present our studies on systematic behaviors of the isospin effect as well as isoscaling features for projectile-like fragments in the framework of statistical abrasion-ablation model. Extraction of the symmetry energy coefficient from the isoscaling parameters will also be investigated. 


\section{Model description}

The statistical abrasion-ablation model can describe the isotopic distribution well [7]. In the SAA model, the nuclear reaction is described as two stages which occur in two distinctly different time scales. The first abrasion stage is fragmentation reaction which describes the production of the pre-fragment with certain amount excitation energy through the independent nucleon-nucleon collisions in the overlap zone of the colliding nuclei. The collisions are described by a picture of interacting tubes. Assuming a binomial distribution for the absorbed projectile neutrons and protons in the interaction of a specific pair of tubes, the distributions of the total abraded neutrons and protons are determined. For an infinitesimal tube in the projectile, the transmission probabilities for neutrons (protons) at a given impact parameter $b$ are calculated by [7]

$$
t_{\mathrm{k}}(\vec{r}-\vec{b})=\exp \left\{-\left[D_{\mathrm{n}}^{\mathrm{T}}(\vec{r}-\vec{b}) \sigma_{\mathrm{nk}}+D_{\mathrm{p}}^{\mathrm{T}}(\vec{r}-\vec{b}) \sigma_{\mathrm{pk}}\right]\right\},
$$

where $D^{\mathrm{T}}$ is thickness function of the target, which is normalized by $\int d^{2} r D_{\mathrm{n}}^{\mathrm{T}}=N^{\mathrm{T}}$ and $\int d^{2} r D_{\mathrm{p}}^{\mathrm{T}}=Z^{\mathrm{T}}$ with $N^{\mathrm{T}}$ and $Z^{\mathrm{T}}$ referring to the neutron and proton number in the target respectively, the vectors $\vec{r}$ and $\vec{b}$ are defined in the plane perpendicular to beam, and $\sigma_{\mathrm{k}^{\prime} \mathrm{k}}$ is the free nucleon-nucleon cross sections $\left(\mathrm{k}^{\prime}, \mathrm{k}=\mathrm{n}\right.$ for neutron and $\mathrm{k}^{\prime}$, $\mathrm{k}=\mathrm{p}$ for proton). The thickness function of the target is given by

$$
D_{\mathrm{k}}^{\mathrm{T}}(r)=\int_{-\infty}^{+\infty} d z \rho_{\mathrm{k}}\left(\left(r^{2}+z^{2}\right)^{1 / 2}\right)
$$

with $\rho_{\mathrm{k}}$ being the neutron (proton) density distribution of the target. So the average abraded mass at a given impact parameter $b$ is calculated by the expression

$$
\begin{aligned}
\langle\Delta A(b)\rangle= & \int d^{2} r D_{\mathrm{n}}^{\mathrm{P}}(r)\left[1-t_{\mathrm{n}}(\vec{r}-\vec{b})\right] \\
& +\int d^{2} r D_{\mathrm{p}}^{\mathrm{P}}(r)\left[1-t_{\mathrm{p}}(\vec{r}-\vec{b})\right] .
\end{aligned}
$$

The second stage is the evaporation process in which the system reorganizes due to excitation. It deexcites and thermalizes by the cascade evaporation of light particles using the conventional statistical model [35]. The excitation energy for projectile spectator is estimated by a simple relation of $E^{*}=13.3\langle\Delta A(b)\rangle \mathrm{MeV}$ where 13.3 is a mean excitation energy for an abraded nucleon from the initial projectile. This excitation energy was given by the statistical hole-energy model as described in Ref. [35]. After the evaporation stage, we can obtain the final fragments which are comparable to the experimental data. By introducing in-medium nucleon-nucleon cross section and optimizing computational method given in Ref. [36, 18, 19, 37], it can give a good agreement with the experimental isotopic distributions [18, 19, 37]. Comparison of the SAA model calculations with the experimental isotopic distributions for $Z=30-32$ from $44 A \mathrm{MeV}{ }^{86} \mathrm{Kr}+{ }^{27} \mathrm{Al}$ [38] and $Z=43-45$ from $790 A \mathrm{MeV}{ }^{129} \mathrm{Xe}+{ }^{27} \mathrm{Al}$ [39] is shown in Fig. 1. The results shown in this figure and all the following figures are referring the final fragments after the evaporation stage. For ${ }^{86} \mathrm{Kr}$, all isotopic distributions are normalized

by the same factor in order to compare with the experimental yields. For ${ }^{129} \mathrm{Xe}$, the calculated production cross sections are compared with the data directly. From this 


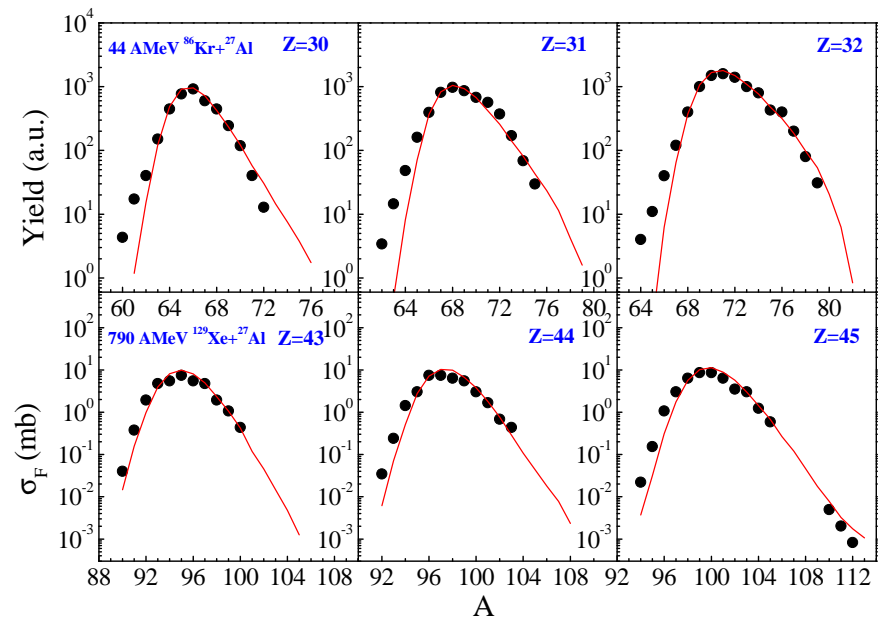

Figure 1. Comparison of isotopic distributions between the SAA model and the data. The isotopic distributions for selected charge numbers from $44 A \mathrm{MeV}{ }^{86} \mathrm{Kr}+{ }^{27} \mathrm{Al}$ (upper panel) and $790 \mathrm{AMeV}{ }^{129} \mathrm{Xe}+{ }^{27} \mathrm{Al}$ (lower panel). The dots are the experimental data taken from Ref. 38 for $44 \mathrm{AMeV}{ }^{86} \mathrm{Kr}+{ }^{27} \mathrm{Al}$ and Ref.[39] for $790 \mathrm{AMeV}$ ${ }^{129} \mathrm{Xe}+{ }^{27} \mathrm{Al}$, the lines are the results calculated by the SAA model.

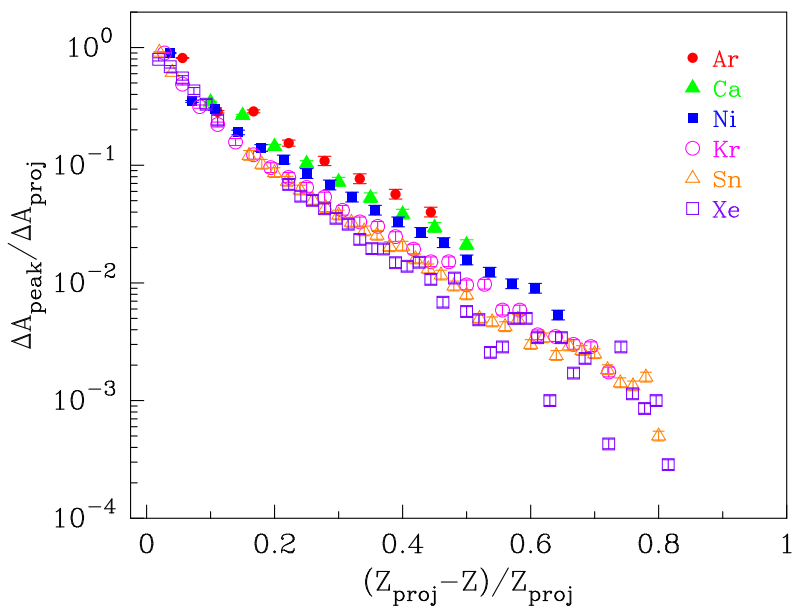

Figure 2. The normalized peak difference $\Delta \mathrm{A}_{\text {peak }} / \Delta \mathrm{A}_{\text {proj }}$ of the fragment isotopic distribution as a function of $\left(Z_{\text {proj }}-Z\right) / Z_{\text {proj. }}$. Different symbols are used for projectiles with different charge number as shown in the legend.

figure, we can see that the SAA model can reproduce the experimental data both at intermediate and high energies quite well. The isospin effect and its disappearance in projectile fragmentation for ${ }^{36,40} \mathrm{Ar}$ at intermediate energies have been predicted by this model and confirmed by the experimental data [19].

\section{Calculations and discussion}

The model predicts that strong isospin effect exists in the isotopic distributions produced by projectiles with same charge number but different mass number [18, 19]. In order 


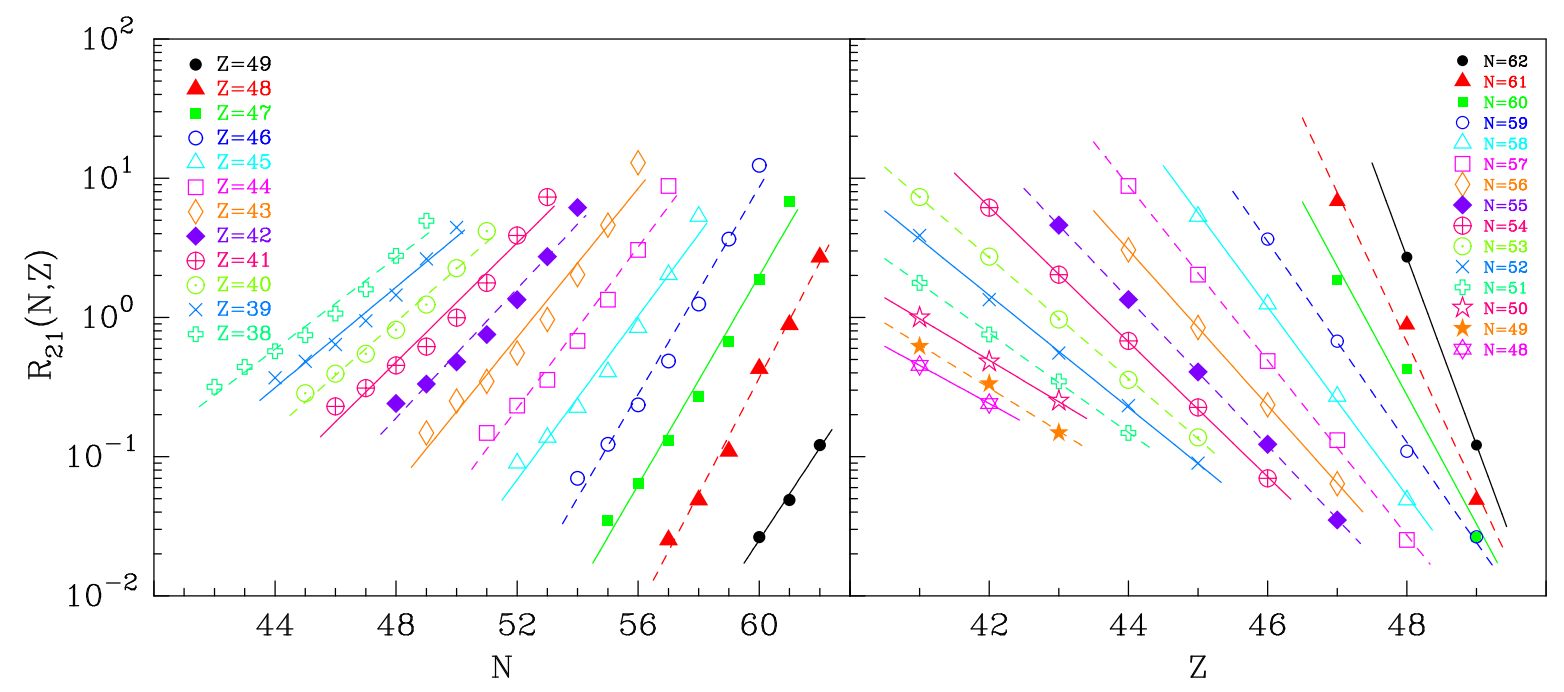

Figure 3. Yield ratios $R_{21}(N, Z)$ of fragments from the reactions of ${ }^{124 / 112} \mathrm{Sn}+{ }^{112} \mathrm{Sn}$ at $60 \mathrm{~A} \mathrm{MeV}$ versus $N$ for the selected isotopes (left panel) and $Z$ for the selected isotones (right panel). Different symbols are used for different isotopes and isotones as shown in the legend. The lines represent the exponential fits. For details see text.

to do a systematic study of the isospin effect in projectile fragmentation, reactions of ${ }^{40 / 36} \mathrm{Ar},{ }^{48 / 40} \mathrm{Ca},{ }^{64 / 58} \mathrm{Ni},{ }^{86 / 78} \mathrm{Kr},{ }^{124 / 112} \mathrm{Sn}$ and ${ }^{129 / 136} \mathrm{Xe}$ on ${ }^{112} \mathrm{Sn}$ at $60 \mathrm{~A} \mathrm{MeV}$ are simulated by the SAA model. Since the isotopic distributions from two projectiles have similar shape but a shift in mass, their peak positions will be one of the most sensitive quantities for the isospin effect. Thus we extract the peak position by Gaussian fit to the fragment isotopic distribution for each charge number $\mathrm{Z}$ as in Ref.[18, 19]. The normalized differences of the peak position from two projectiles $\Delta \mathrm{A}_{\text {peak }} / \Delta \mathrm{A}_{\text {proj }}$ as a function of $\left(Z_{\text {proj }}-Z\right) / Z_{\text {proj }}$ are shown in Fig. 2. Here $\Delta \mathrm{A}_{\text {proj }}$ is the mass number difference between the two projectiles with same charge number and $\Delta \mathrm{A}_{\text {peak }}$ is the peak position difference of the fragment isotopic distribution produced by these two projectiles. $Z_{\text {proj }}$ and $Z$ are the charge number of the projectile and the produced isotopes. $\Delta \mathrm{A}_{\text {peak }} / \Delta \mathrm{A}_{\text {proj }}$ exponentially decreases as the increase of $\left(Z_{\text {proj }}-Z\right) / Z_{\text {proj }}$ which is same as our previous conclusions [19]. The dependence of $\Delta \mathrm{A}_{\text {peak }} / \Delta \mathrm{A}_{\mathrm{proj}}$ on $\left(Z_{\text {proj }}-Z\right) / Z_{\text {proj }}$ shows a very slight difference among different size projectiles.

To study systematic behaviors of the isoscaling phenomena, the yield ratios $R_{21}(N, Z)$ are made using the convention that index 2 refers to the more neutronrich system and index 1 to the less neutron-rich one. As an example, Fig. 3 shows the yield ratios $R_{21}(N, Z)$ as a function of neutron number $N$ for selected isotopes and $Z$ for selected isotones from ${ }^{124 / 112} \mathrm{Sn}+{ }^{112} \mathrm{Sn}$ reactions in log-scale. From this figure, we observe that the ratio for each isotope $Z$ exhibits a remarkable exponential behavior. For each isotope $(Z)$, an exponential function form $C \exp (\alpha N)$ is used to fit the calculated points and the parameters $\alpha$ are obtained for all isotopes. Analogous behavior is observed for each isotone $(N)$, an exponential function form $C^{\prime} \exp (\beta Z)$ is 
used to fit the calculated points and the parameters $\beta$ are obtained for all isotones.

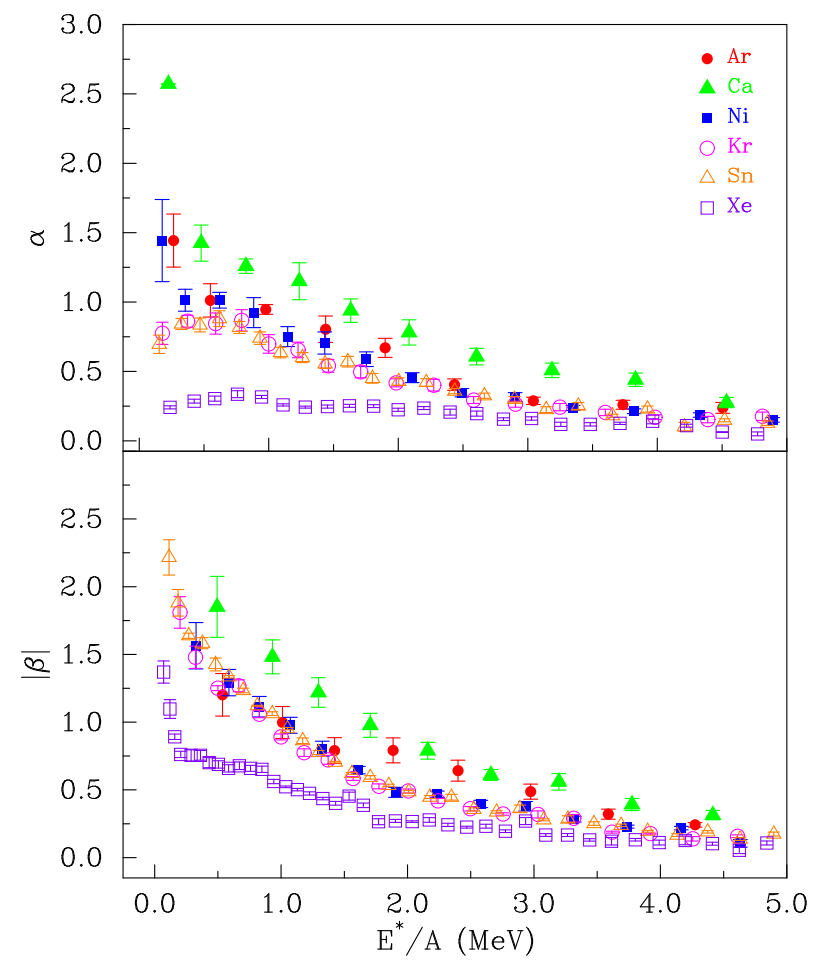

Figure 4. Same as in Fig. 2 but for the isoscaling parameters $\alpha$ (upper panel) and $|\beta|$ (lower panel) as a function of the excitation energy per nucleon.

In Fig. 4, we present the extracted slope parameters $\alpha$ (upper panel) and $|\beta|$ (lower panel) of the exponential fits as a function of the excitation energy per nucleon $\left(E^{*} / A\right)$. Since the excitation energy changes as a function of time in the process of evaporation, the values of $E^{*} / A$ shown here are taken at the beginning of the evaporation stage and the mass of the prefragment is used to calculated $E^{*} / A$. In the model the excitation energy is proportional to the abraded nucleons and can reflect the violence of the collision as the parameter $\left(Z_{\text {proj }}-Z\right) / Z_{\text {proj }}$ shows [19]. In this figure, $\alpha$ and $|\beta|$ show a decreasing trend with the increasing of $E^{*} / A$. This behavior for projectilelike fragments is different with light particles. The isoscaling parameters of light fragments from multifragmentation is almost constant for different isotopes because the excitation energy or temperature is almost same for all light fragments in the process of multifragmentation. The decrease of the isoscaling parameters in our calculations may mainly be attributed to the evaporation effect of the prefragment with different excitation energy as in the disappearance of the isospin effect [18, 19]. The values of $\alpha$ and $|\beta|$ are quite different from different reaction systems due to the different size and isospin of the projectiles.

According to Eq. (2) and (3), $\alpha$ and $\beta$ have a linear dependence on $\Delta\left[\left(\frac{Z}{A}\right)^{2}\right]$ or $\Delta\left[\left(\frac{N}{A}\right)^{2}\right]$. Since this parameter is dependent on the reaction system, we divide $\alpha(\beta)$ by $\Delta\left[\left(\frac{Z}{A}\right)^{2}\right]\left(\Delta\left[\left(\frac{N}{A}\right)^{2}\right]\right)$ to remove the system isospin and size dependence and 


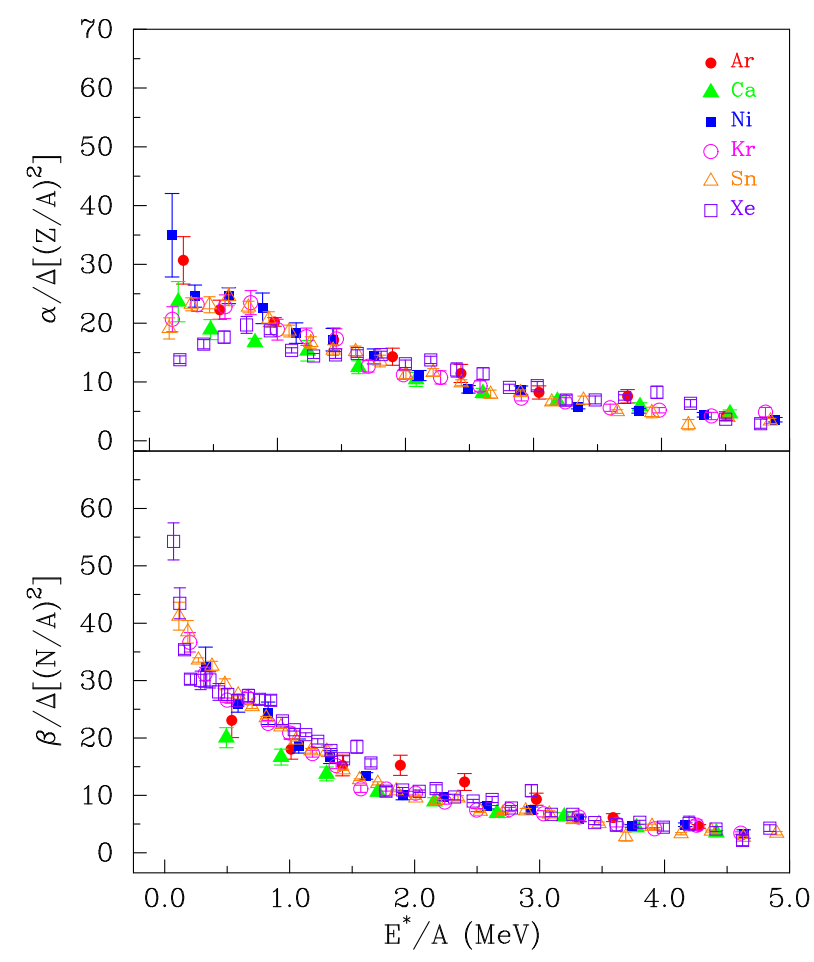

Figure 5. Same as in Fig. 2 but for the reduced isoscaling parameters $\alpha / \Delta\left[\left(\frac{Z}{A}\right)^{2}\right]$ (upper panel) and $\beta / \Delta\left[\left(\frac{N}{A}\right)^{2}\right]$ (lower panel) as a function of the excitation energy per nucleon.

call them reduced isoscaling parameters. The results are given in Fig. 5, After the reduction, $\alpha / \Delta\left[\left(\frac{Z}{A}\right)^{2}\right]\left(\beta / \Delta\left[\left(\frac{N}{A}\right)^{2}\right]\right)$ of different reaction systems demonstrate almost same dependence with $E^{*} / A$. Eq. (2) and (3) are deduced from the grand-canonical limit for multifragmentation of hot source. For projectile-like fragments, the same behavior is observed in the SAA model. In this sense, the reduced isoscaling parameters $\alpha / \Delta\left[\left(\frac{Z}{A}\right)^{2}\right]$ $\left(\beta / \Delta\left[\left(\frac{N}{A}\right)^{2}\right]\right)$ may be used as a sensitive observable for measuring the excitation extent of projectile-like fragments during the collisions without system size dependence.

From above discussions, we found that $\Delta \mathrm{A}_{\text {peak }} / \Delta \mathrm{A}_{\text {proj }}$ and $\alpha / \Delta\left[\left(\frac{Z}{A}\right)^{2}\right]$ $\left(\beta / \Delta\left[\left(\frac{N}{A}\right)^{2}\right]\right)$ decrease with $\left(Z_{\text {proj }}-Z\right) / Z_{\text {proj }}$ or the excitation energy per nucleon. But the later one decreases slower than the first one. It means that $\alpha / \Delta\left[\left(\frac{Z}{A}\right)^{2}\right]\left(\beta / \Delta\left[\left(\frac{N}{A}\right)^{2}\right]\right)$ is more sensitive to the isospin effect of the projectiles. Since $\alpha / \Delta\left[\left(\frac{Z}{A}\right)^{2}\right]\left(\beta / \Delta\left[\left(\frac{N}{A}\right)^{2}\right]\right)$ is related to $\frac{C_{\mathrm{Sym}}}{T}$ as in Eq. (2) and (3), it can be used as an observable to study the excitation and asymmetric nuclear equation of state in heavy ion collisions.

If we use the Fermi-gas relationship between excitation energy per nucleon and temperature $E^{*} / A=\frac{1}{a} T^{2}$ to calculate $T$ tentatively, with the inverse level density parameter $a=8-13$ (in our calculation $a=10$ is used), the symmetry energy coefficient $\left(C_{\text {Sym }}\right)$ could be extracted. Results extracted from $\alpha / \Delta\left[\left(\frac{Z}{A}\right)^{2}\right]$ and $\beta / \Delta\left[\left(\frac{N}{A}\right)^{2}\right]$ are shown in Fig. 6. For $E^{*} / A$ around $1 \mathrm{MeV}$, the symmetry energy coefficients from $\alpha$ and $\beta$ are around $15 \mathrm{MeV}$. These values are a little lower than the standard value $C_{\mathrm{sym}}=25 \mathrm{MeV}$ 


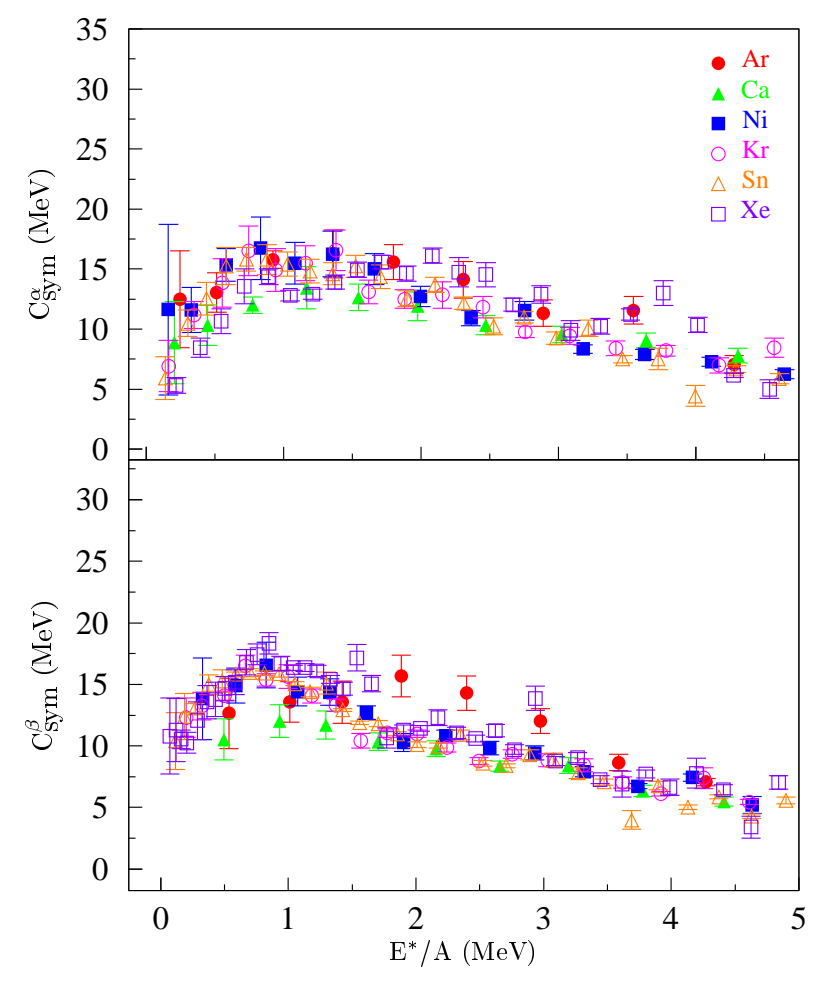

Figure 6. Same as in Fig. 2 but for the extracted symmetry energy coefficient from $\alpha$ $\left(C_{\mathrm{sym}}^{\alpha}\right.$, upper panel $)$ and $\beta\left(C_{\mathrm{sym}}^{\beta}\right.$, lower panel $)$ as a function of the excitation energy per nucleon.

in liquid drop model [23], but it seems consistent with the extracted results from the experimental data by A. Le Févre et al. [28]. The obtained $C_{\text {sym }}$ is not a constant and decreases with the increase of $E^{*} / A$. Similar dependence was also observed in

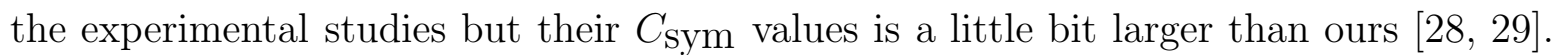
It should be pointed out that the experimental data are taken at different incident energies (300 A MeV and $600 A \mathrm{MeV}$ in Ref. [28], around $25 A \mathrm{MeV}$ in Ref. [29]). Our calculations are performed at $60 \mathrm{~A} \mathrm{MeV}$, but we have found that there is almost no incident energy dependence for the isoscaling parameters in our model. In our results, the symmetry energy coefficient decreases quickly when $E^{*} / A$ is less than 1 $\mathrm{MeV}$. This may stem from the increase of the inverse level density parameter at low excitation energy [40]. Of course, in this low $E^{*} / A$ range there is very few theoretical and experimental data up to now and more researches are necessary. Experimentally it may be difficult to extract $E^{*} / A$. As we have mentioned previously that the parameter $\left(Z_{\text {proj }}-Z\right) / Z_{\text {proj }}$ can reflect the violence of nuclear collision and is approximately proportional to $E^{*} / A$ in not very central collisions. Actually quite similar dependence as in Fig. 4,6 is seem if $\left(Z_{\text {proj }}-Z\right) / Z_{\text {proj }}$ is used instead of $E^{*} / A$. Thus we can also study the dependence of the isoscaling parameter and symmetry energy coefficient with $\left(Z_{\text {proj }}-Z\right) / Z_{\text {proj }}$ experimentally when there is no $E^{*} / A$ data.

However, some cautions should be reminded. In the SAA model, the symmetry 
energy term is not taken into account explicitly. But a similar analysis of isoscaling as in the statistical multifragmentation model could be done since there exists different isotopic and isotonic distributions between two systems. Of course, the effect of symmetry energy term should have been reflected implicitly in the assumption of the abrasion and also evaporation stages in the SAA model. Thus a clear isoscaling behavior is observed for projectile-like fragments in the this work and the symmetry energy coefficient extracted based on Eq. (2) and (3) is consistent with the experimental data [28, 29]. The present calculation could provide some useful information for further experimental and theoretical investigations on the isoscaling of projectile-like fragments.

\section{Summary}

In summary, systematic behaviors of the isospin effect and isoscaling of projectile-like fragments from ${ }^{40 / 36} \mathrm{Ar},{ }^{48 / 40} \mathrm{Ca},{ }^{64 / 58} \mathrm{Ni},{ }^{86 / 78} \mathrm{Kr},{ }^{124 / 112} \mathrm{Sn}$ and ${ }^{129 / 136} \mathrm{Xe}$ on ${ }^{112} \mathrm{Sn}$ at $60 \mathrm{~A} \mathrm{MeV}$ have been studied by a modified statistical abrasion-ablation model. The

normalized peak differences $\Delta \mathrm{A}_{\text {peak }} / \Delta \mathrm{A}_{\text {proj }}$ for different reaction systems show similar dependence with the parameter $\left(Z_{\text {proj }}-Z\right) / Z_{\text {proj }}$. The isoscaling parameters $\alpha$ and $\beta$ are extracted for the produced isotopes and isotones, and they show different values for different systems. However, the reduced isoscaling parameters $\alpha / \Delta\left[\left(\frac{Z}{A}\right)^{2}\right]$ and $\beta / \Delta\left[\left(\frac{N}{A}\right)^{2}\right]$ show almost same dependence with $E^{*} / A$ for different systems. Assuming a Fermi-gas behavior, the symmetry energy coefficients are tentatively extracted from $\alpha$ and $\beta$ and it seems that the results are consistent with the experimental data.

\section{Acknowledgments}

This work was partially supported by the National Natural Science Foundation of China (NNSFC) under Grant No. 10405032, 10535010, 10405033 and 10475108, Shanghai Development Foundation for Science and Technology under contract No. 06QA14062, 06JC14082 and 05XD14021, the Major State Basic Research Development Program in China under Contract No. 2007CB815004 and the Knowledge Innovation Project of Chinese Academy of Sciences under Grant No. KJCX3.SYW.N2.

\section{References}

[1] J. D. Bowman, W. J. Swiatecki, C. F. Tang, Lawrence Berkeley Laboratory Report No. LBL-2908 (1973).

[2] J. Hüfner, K. Shäfer, B. Schürmann, Phys. Rev. C 12, 1888 (1975).

[3] J. Gosset et al., Phys. Rev. C 16, 629 (1977).

[4] M. J. Morrissey et al., Phys. Rev. C 18, 1267 (1978).

[5] R. Dayras et al., Nucl. Phys. A 460, 299 (1986).

[6] A. Bonasera, M. di Toro, C. Grégoire, Nucl. Phys. A 463, 653 (1987).

[7] T. Brohm, K.-H. Schmidt, Nucl. Phys. A 569, 821 (1994).

[8] I. Tanihata et al., Phys. Rev. Lett. 55, 2676 (1985); Phys. Lett. B 160, 380 (1985).

[9] I. Tanihata et al., Phys. Lett. B 206, 592 (1988). 
[10] M. L. Miller et al., Phys. Rev. Lett. 82, 1399 (1999).

[11] J. F. Dempsey et al., Phys. Rev. C 54, 1710 (1996).

[12] B. A. Li et al., Phys. Rev. Lett. 76, 4492 (1996).

[13] B. A. Li, C. M. Ko, W. Bauer, Int. J. Mod. Phys. E 7, 147 (1998).

[14] R. Pak et al., Phys. Rev. Lett. 78, 1022 (1997); 1026 (1997).

[15] S. Kumar et al., Phys. Rev. C 58, 3494 (1998).

[16] H. Müller, B. D. Serot, Phys. Rev. C 52, 2072 (1995).

[17] Y. G. Ma et al., Phys. Rev. C 60, 024607 (1999).

[18] D. Q. Fang et al., Phys. Rev. C 61, 044610 (2000); High Energy Phys. Nucl. Phys. 24, 69 (2000) (in Chinese).

[19] D. Q. Fang et al., Eur. Phys. J. A 10, 0381 (2001).

[20] M. B. Tsang et al., Phys. Rev. Lett. 86, 5023 (2001).

[21] M. B. Tsang et al., Phys. Rev. C 64, 041603 (2001).

[22] M. B. Tsang et al., Phys. Rev. C 64, 054615 (2001).

[23] A. S. Botvina, O. V. Lozhkin, W. Trautmann, Phys. Rev. C 65, 044610 (2002).

[24] V. Baran et al., Phys. Rep. 410, 335 (2005).

[25] Y. G. Ma, W. Q. Shen, Nucl. Sci. Tech. 15, 4 (2004).

[26] Y. G. Ma et al., Phys. Rev. C 69, 064610 (2004).

[27] W. D. Tian et al., Chin. Phys. Lett. 22, 306 (2005).

[28] A. Le Fèvre et al., Phys. Rev. Lett. 94, 162701 (2005).

[29] G. A. Souliotis et al., Phys. Rev. C 73, 024606 (2006).

[30] G. A. Souliotis et al., Phys. Rev. C 68, 024605 (2003).

[31] W. A. Friedman, Phys. Rev. C 69, 031601(R) (2004).

[32] M. Veselsky, G. A. Souliotis, M. Jandel, Phys. Rev. C 69, 044607 (2004).

[33] K. Wang et al., Chin. Phys. Lett. 22, 53 (2005).

[34] Y. G. Ma et al., Phys. Rev. C 72, 064603 (2005).

[35] J.-J. Gaimard, K.-H. Schmidt, Nucl. Phys. A 531, 709 (1991).

[36] X. Z. Cai et al., Phys. Rev. C 58, 572 (1998).

[37] C. Zhong et al., High Energ. Phys. Nucl. 27, 39 (2003) (in Chinese).

[38] D. Bazin et al., Nucl. Phys. A 515, 349 (1990).

[39] J. Reinhold et al., Phys. Rev. C 58, 247 (1998).

[40] J. B. Natowitz et al., Phys. Rev. C 65, 034618 (2002). 